\title{
CORRECTION
}

Series A

\section{Correction to: Approximations and generalized Newton methods}

\author{
Diethard Klatte ${ }^{1}\left[\right.$ B $\cdot$ Bernd Kummer ${ }^{2}$
}

Published online: 25 October 2019

(อ) Springer-Verlag GmbH Germany, part of Springer Nature and Mathematical Optimization Society 2019

\section{Correction to: Math. Program., Ser. B (2018) 168:673-716 https://doi.org/10.1007/s10107-017-1194-8}

In Subsection 3.2 of the paper [5], we studied the interrelations between semismoothness (in the sense of Qi, Sun [7]), approximation by Newton maps (in the sense of $[4,6]$ ) and directional differentiability. In a private communication [3], Helmut Gfrerer pointed out that Theorem 6 in [5] is incorrect, and he created a counterexample showing that Proposition 2 in [5] is false.

First we present the correct statement of [5, Theorem 6]. Suppose that $f$ is a locally Lipschitz function from $\mathbb{R}^{n}$ to $\mathbb{R}^{m}$ (briefly $f \in C^{0,1}\left(\mathbb{R}^{n}, \mathbb{R}^{m}\right)$ ). Let $f^{\prime}(\bar{x} ; u$ ) be the standard directional derivative of $f$ at $\bar{x}$ in direction $u$, and denote by $\partial^{C L} f(\bar{x})$ Clarke's generalized Jacobian [1,2] of $f$ at $\bar{x}$. For the definitions of Newton maps and semismoothness, we refer to [5].

Theorem ([5, Theorem 6] corrected). $f$ is semismooth at $\bar{x}$ if and only if

(i) $\partial^{C L} f$ is a Newton map for $f$ at $\bar{x}$, and

(ii) $f^{\prime}(\bar{x} ; u)$ exists for each $u$.

Indeed, by [7, Prop. $2.1 \&$ Thm. 2.3], semismoothness of $f$ at $\bar{x}$ implies both (i) and (ii). On the other hand, the if-direction of the theorem follows from [7, Thm. 2.3], by taking $f^{\prime}(\bar{x} ; u)=f(\bar{x}+u)-f(\bar{x})+o(u)$ under (ii) into account (for the latter see, e.g., [4, Lemma A2], [8]).

The original article can be found online at https://doi.org/10.1007/s10107-017-1194-8.

Diethard Klatte

diethard.klatte@uzh.ch

Bernd Kummer

kummer@math.hu-berlin.de

1 IBW, Universität Zürich, Moussonstrasse 15, 8044 Zurich, Switzerland

2 Institut für Mathematik, Humboldt-Universität zu Berlin, Unter den Linden 6, 10099 Berlin, Germany 
The original version of [5, Theorem 6] included the incorrect statement that (ii) follows from (i), while [5, Proposition 2] claimed that even a Newton-map property weaker than (i) implies (ii). Note that in the proof of Proposition 2 in [5], the estimate [... $\geq 2$ if $h_{k} \geq 0$ ] on line 6 of page 690 is false.

We finish this corrigendum by Gfrerer's counterexample [3]: it gives a function $f \in C^{0,1}(\mathbb{R}, \mathbb{R})$ which satisfies the Newton-map property (i), but is not directionally differentiable and hence not semismooth.

Example Consider two real sequences $a_{k} \downarrow 0, b_{k} \downarrow 0$ given by

$$
a_{1}:=1, b_{k}:=e^{-2 k} a_{k}, a_{k+1}:=e^{-2 k} b_{k}, k \geq 1,
$$

and the function $f: \mathbb{R} \rightarrow \mathbb{R}$ defined by

$$
f(x):= \begin{cases}x & \text { if } x \geq 1 \\ -x+\frac{1}{k} x \ln \frac{x}{b_{k}} & \text { if } x \in\left[b_{k}, a_{k}\right), k \geq 1, \\ x-\frac{1}{k} x \ln \frac{x}{a_{k+1}} & \text { if } x \in\left[a_{k+1}, b_{k}\right), k \geq 1 \\ 0 & \text { if } x \leq 0 .\end{cases}
$$

$f$ is continuous, since for $k \geq 1$,

$\lim _{x \uparrow a_{k}} f(x)=\lim _{x \uparrow a_{k}}\left(-x+\frac{1}{k} x \ln \frac{x}{b_{k}}\right)=-a_{k}+\frac{1}{k} a_{k} \ln \frac{a_{k}}{b_{k}}=-a_{k}+\frac{1}{k} a_{k} 2 k=a_{k}=f\left(a_{k}\right)$,
$\lim _{x \uparrow b_{k}} f(x)=\lim _{x \uparrow b_{k}}\left(x-\frac{1}{k} x \ln \frac{x}{a_{k+1}}\right)=b_{k}-\frac{1}{k} b_{k} \ln \frac{b_{k}}{a_{k+1}}=b_{k}-\frac{1}{k} b_{k} 2 k=-b_{k}=f\left(b_{k}\right)$,

while $\lim _{x \downarrow 0} f(x)=0=f(0)$ because of

$$
\begin{aligned}
& -x \leq f(x) \leq-x+\frac{1}{k} x \ln \frac{a_{k}}{b_{k}}=-x+\frac{1}{k} x 2 k=x, x \in\left[b_{k}, a_{k}\right), \\
& -x=x-\frac{1}{k} x 2 k=x-\frac{1}{k} x \ln \frac{b_{k}}{a_{k+1}} \leq f(x) \leq x, x \in\left[a_{k+1}, b_{k}\right) .
\end{aligned}
$$

$f$ is continuously differentiable except for the points $0, a_{k}, b_{k}(k \geq 1)$, with derivative

$$
f^{\prime}(x)= \begin{cases}1 & \text { if } x>1 \\ \frac{f(x)}{x}+\frac{1}{k} & \text { if } x \in\left(b_{k}, a_{k}\right) \\ \frac{f(x)}{x}-\frac{1}{k} & \text { if } x \in\left(a_{k+1}, b_{k}\right) \\ 0 & \text { if } x<0\end{cases}
$$


Then we have

$$
\partial^{C L} f(x)= \begin{cases}{[1,2]} & \text { if } x=a_{1}, \\ {\left[1-\frac{1}{k}, 1+\frac{1}{k+1}\right]} & \text { if } x=a_{k+1}, k \geq 1, \\ {\left[-1-\frac{1}{k},-1+\frac{1}{k}\right]} & \text { if } x=b_{k}, k \geq 1, \\ {[-1,1]} & \text { if } x=0, \\ f^{\prime}(x) \in[-2,2] & \text { else }\end{cases}
$$

and Lipschitz continuity of $f$. Thus, for any $x<1$ and any $A \in \partial^{C L} f(x)$, one has

$$
|f(x)-f(0)-A(x-0)| \begin{cases}=\frac{x}{k} & \text { if } x \in\left(a_{k+1}, a_{k}\right) \backslash\left\{b_{k}\right\}, k \geq 1 \\ \leq \frac{x}{k} & \text { if } x=b_{k} \text { or } x=a_{k+1}, k \geq 1 \\ =0 & \text { if } x \leq 0 .\end{cases}
$$

Hence, by definition, $\partial^{C L} f$ is a Newton map for $f$ at $\bar{x}=0$. On the other hand,

$$
\lim _{k \rightarrow \infty} \frac{f\left(a_{k}\right)-f(0)}{a_{k}-0}=1, \quad \lim _{k \rightarrow \infty} \frac{f\left(b_{k}\right)-f(0)}{b_{k}-0}=-1,
$$

and so $f^{\prime}(0 ; 1)$ does not exist. Consequently, $f$ is not semismooth at $\bar{x}=0$.

\section{References}

1. Clarke, F.H.: On the inverse function theorem. Pac. J. Math. 64, 97-102 (1976)

2. Clarke, F.H.: Optimization and Nonsmooth Analysis. Wiley, New York (1983)

3. Gfrerer, H.: Private Communication (2019)

4. Klatte, D., Kummer, B.: Nonsmooth Equations in Optimization-Regularity, Calculus, Methods and Applications. Kluwer Academic Publication, Dordrecht (2002)

5. Klatte, D., Kummer, B.: Approximations and generalized Newton methods. Math. Program. Ser. B 168, 673-716 (2018)

6. Kummer, B.: Newton's method based on generalized derivatives for nonsmooth functions: convergence analysis. In: Oettli, W., Pallaschke, D. (eds.) Advances in Optimization, pp. 171-194. Springer, Berlin (1992)

7. Qi, L., Sun, L.: A nonsmooth version of Newton's method. Math. Program. 58, 353-367 (1993)

8. Shapiro, A.: On concepts of directional differentiability. J. Optim. Theory Appl. 66, 477-487 (1990)

Publisher's Note Springer Nature remains neutral with regard to jurisdictional claims in published maps and institutional affiliations. 